\title{
Year in Review 2014: Airway Clearance
}

\author{
Shawna L Strickland PhD RRT-NPS ACCS AE-C FAARC
}

\author{
Introduction \\ American Association for Respiratory Care Clinical Practice Guideline \\ Chest Physical Therapy \\ HFCWC \\ IPV \\ Mechanical Insufflation-Exsufflation \\ PEP \\ Summary
}

\begin{abstract}
The respiratory therapist has had integrated adjuncts to improve mucus clearance for decades. However, there is a lack of literature describing the impact of these interventions on specific patient populations, resulting in an inability to make recommendations about the use of devices and techniques. The purpose of this article is to review recent literature regarding airway clearance therapies in a manner that is most likely to have interest to the readers of Respiratory Care. Key words: airway clearance; chest physical therapy; high-frequency chest-wall compression; intrapulmonary ventilation; mechanical insufflation-exsufflation; positive expiratory pressure. [Respir Care 2015;60(4):603-605. () 2015 Daedalus Enterprises]
\end{abstract}

\section{Introduction}

The use of airway clearance techniques is often based on anecdotal evidence or historical practice rather than on evidence. In truth, there is a significant lack of high-level evidence for airway clearance techniques. ${ }^{1}$ Recent research has focused on a variety of airway clearance interventions, including chest physical therapy, high-frequency chest-

Dr Strickland is affiliated with the American Association for Respiratory Care, Irving, Texas.

Dr Strickland presented a version of this paper at the Year in Review 2014 of the AARC Congress 2014, held December 9-12, 2014, in Las Vegas, Nevada.

Dr Strickland has disclosed no conflicts of interest.

Correspondence: Shawna L Strickland PhD RRT-NPS ACCS AE-C FAARC, American Association for Respiratory Care, 9425 North MacArthur Boulevard, Suite 100, Irving, TX 75063. E-mail: shawna.strickland@aarc.org.

DOI: $10.4187 /$ respcare.04095 wall compression (HFCWC), intrapulmonary ventilation (IPV), mechanical insufflation-exsufflation, and positive expiratory pressure (PEP), in a variety of patient populations.

\section{American Association for Respiratory Care Clinical Practice Guideline}

In late 2013, the American Association for Respiratory Care (AARC) published "AARC clinical practice guideline: effectiveness of non-pharmacologic airway clearance therapies in hospitalized patients." guideline put forth 9 recommendations for airway clearance techniques in 3 different clinical scenarios: hospitalized adult and pediatric patients without cystic fibrosis; adult and pediatric patients with neuromuscular disease, respiratory muscle weakness, or impaired cough; and postoperative adult and pediatric patients. Although supported by low-level evidence, the recommendations identified that airway clearance techniques are generally not indicated for routine use in all patient populations. The lack of evidence 
is the driving force behind an inability to recommend many of the airway clearance techniques in specific situations. ${ }^{1}$

\section{Chest Physical Therapy}

Considering that the purported benefit of chest physical therapy is a reduction in retained secretions, it has been hypothesized, although not proven, that a patient's ventilation and oxygenation will improve as a result. ${ }^{1-3}$ In a well-powered study investigating the effectiveness of chest physical therapy in mechanically ventilated subjects diagnosed with sepsis, a statistically significant short-term improvement in oxygenation and ventilation was observed. ${ }^{4}$ The researchers' conclusion that chest physical therapy may produce immediate effects of improved oxygenation $(P<.02)$ and lactate levels $(P<.001)$ is tempered by the lack of discussion as to whether the improvement is sustainable or temporary and by the number of uncontrolled variables and the immediate timeframe of the post-intervention measurements. Ultimately, existing evidence does not support the routine use of chest physical therapy. Even in the presence of symptomatic secretion retention, chest physical therapy is recommended only if clinical indicators of effectiveness are demonstrated and if the patient tolerates the procedure. ${ }^{1}$

\section{HFCWC}

Typical applications of HFCWC are limited to patients with thicker secretions, although there is no clear evidence to support whether this intervention is more or less effective than any other airway clearance technique..$^{5}$ In patient populations in which generating an effective cough is problematic, the role of HFCWC is unclear. Fitzgerald et $\mathrm{al}^{6}$ hypothesized that adherence to a long-term airway clearance regime including HFCWC in the home improves morbidity due to respiratory infections and reduces the number of hospitalizations for children with neurological impairment who are unable to generate an effective cough. Although the study demonstrated a reduction in the number of hospitalizations after initiation of HFCWC, the outcomes cannot be generalized due to small sample size $(N=7)$, minimum study power, and lack of randomization.

In another study focusing on the use of HFCWC in subjects unable to generate an effective cough, HFCWC was applied after extubation to prevent pulmonary complications and subsequent re-intubation. ${ }^{7}$ Subjects included in the study sample had been intubated for $21 \mathrm{~d}$ or more and demonstrated a small improvement in chest radiographs compared with subjects who did not receive HFCWC $(P=.02)$. However, the researchers noted no improvement in weaning success rates, oxygenation, anxiety levels, perceived dyspnea, or ICU stay. Due to a lack of evidence, HFCWC is not recommended for routine therapy. ${ }^{1}$

\section{IPV}

In an effort to determine the efficacy of IPV in the COPD population, Testa et $\mathrm{l}^{8}$ added IPV to the existing pulmonary regimens of 20 subjects with COPD. The existing regimens consisted of combinations of pulmonary rehabilitation, chest physical therapy, PEP, and cough techniques. Although none of the subjects suffered adverse reactions or harm from the IPV, post-intervention measures of oxygenation, pulmonary mechanics, and dyspnea scores did not differ from pre-intervention measures, leading to the conclusion that IPV does not provide additional benefit in this patient population. The evidence for IPV is insufficient to make claims for safety or efficacy. ${ }^{1}$

\section{Mechanical Insufflation-Exsufflation}

Although the evidence is weak, cough-assist techniques, including mechanical insufflation-exsufflation, have been recommended for patients with neuromuscular disease, in particular when the cough peak flow is $<270 \mathrm{~L} / \mathrm{min}^{1}{ }^{1}$ Lacombe et $\mathrm{al}^{9}$ discovered, however, that mechanical insufflation-exsufflation alone may not be enough to produce adequate airway clearance. As an independent airway clearance technique, mechanical insufflation-exsufflation generates a lower cough peak flow than it does when paired with manually assisted cough and also intermittent positive-pressure breathing paired with manually assisted cough. ${ }^{9}$ However, evidence on both short-term and long-term patient outcomes and adverse effects of mechanical insufflation-exsufflation is significantly lacking, which limits the ability to draw conclusions about its safety or efficacy in this population. ${ }^{10}$

\section{PEP}

Although PEP (with and without oscillation) has been used for several years, questions remain as to which setting is appropriate for optimum results and whether the device may affect other therapies. Specifically studying the Acapella device, Mueller et al ${ }^{11}$ focused on determining which setting produced optimum frequency with higher vibratory amplitudes. Interestingly, the Acapella product line has 3 different models, and all 3 models performed differently at the same setting, indicating that it is important for the respiratory therapist to understand the differences not only among different manufacturers but also among the different devices produced by the same manufacturer.

When administering PEP therapy in line with nebulized medication, placement of the device within the nebulizer circuit was noted to significantly reduce the mass median aerodynamic diameter of the aerosol particles, which impacts how much of the medication is delivered to the lower 
airway. ${ }^{12}$ The degree to which the mass median aerodynamic diameter is altered is dependent upon type of PEP device, the presence of oscillations or vibrations, and the type of nebulizer. Although the convenience of delivering 2 therapies concurrently is tempting, the reality is that aerosol delivery may be far less effective than when delivered independent of the PEP device, again emphasizing the importance of understanding differences among devices. ${ }^{12}$

The routine use of PEP for patients with COPD is not recommended but may be an option for those patients with COPD and symptomatic secretion retention. ${ }^{1}$ Osadnik et al ${ }^{13}$ conducted a randomized controlled trial to study the effect of PEP added to the usual care of hospitalized subjects with COPD exacerbations. They found that adding PEP to the hospitalized pulmonary regime did not improve symptoms, quality of life, or incidence of future exacerbations. Indeed, the intervention group demonstrated more COPD exacerbations, more respiratory-related hospitalizations, and more hospitalized days compared with the control group, reinforcing the position that PEP is not recommended for routine use in patients with COPD.

Although not a population addressed in the 2013 AARC clinical practice guideline, patients with cystic fibrosis have been identified as candidates for PEP therapy. However, there has been dispute as to whether PEP with oscillations or vibrations is more beneficial than other airway clearance therapies, including PEP without oscillations or vibrations. A meta-analysis evaluated oscillatory or vibratory PEP devices compared with standard PEP, active cycle of breathing, chest physical therapy, and exercise and identified several limitations to generalizing the results of these studies. ${ }^{5}$ The key finding was that there is no evidence that devices that deliver PEP with oscillations or vibrations are better than any other form of airway clearance technique.

\section{Summary}

Although the research varied by intervention, outcomes measured, and patient population, the theme that resonates is that the evidence does not support the routine use of airway clearance therapies. ${ }^{1}$ Further research must use randomized control trials when ethically supported to provide a strong foundation for future recommendations. It is plausible that much of the airway clearance therapy currently ordered in acute care hospitals may be unnecessary and therefore exerts an undue burden on both the patient and the heath-care system. The respiratory therapist's role in recommending airway clearance therapies is centered on being an advocate for the patient and identifying appropriate airway clearance techniques based on clinical indications, solid benefit-burden analysis, and cost analysis while also integrating patient preference, comfort, and likelihood of long-term adherence.

\section{REFERENCES}

1. Strickland SL, Rubin BK, Drescher GS, Haas CF, O'Malley CA, Volsko TA, et al. AARC clinical practice guideline: effectiveness of non-pharmacologic airway clearance therapies in hospitalized patients. Respir Care 2013;58(12):2187-2193.

2. Main E, Prasad A, van der Schans CP. Conventional chest physiotherapy compared to other airway clearance techniques for cystic fibrosis. Cochrane Database Syst Rev 2005;(1):CD002011.

3. Holody B, Goldberg HS. The effect of mechanical vibration physiotherapy on arterial oxygenation in acutely ill patients with atelectasis or pneumonia. Am Rev Respir Dis 1981;124(4):372-375.

4. dos Santos RS, Donadio MV, da Silva GV, Blattner CN, Melo DA, Nunes FB, et al. Immediate effects of chest physiotherapy on hemodynamic, metabolic, and oxidative stress parameters in subjects with septic shock. Respir Care 2014;59(9):1398-1403.

5. Morrison L, Agnew J. Oscillating devices for airway clearance in people with cystic fibrosis. Cochrane Database Syst Rev 2014;(7): CD006842.

6. Fitzgerald K, Dugre J, Pagala S, Homel P, Marcus M, Kazachkov M. High-frequency chest wall compression therapy in neurologically impaired children. Respir Care 2014;59(1):107-112.

7. Huang WC, Wu PC, Chen CJ, Cheng YA, Shis SJ, Chen HC, Wu $\mathrm{CL}$. High-frequency chest wall oscillation in prolonged mechanical ventilation patients: a randomized control trial. Clin Respir J 2014 [Epub ahead of print] doi: 10.1111/crj.12212.

8. Testa A, Galeri S, Villafañe JH, Corbellini C, Pillastrini P, Negrini S. Efficacy of short-term intrapulmonary percussive ventilation in patients with chronic obstructive pulmonary disease. Disabil Rehabil 2014 [Epub ahead of print] doi: 10.3109/09638288.2014.948130.

9. Lacombe M, Del Amo Castrillo L, Boré A, Chapeau D, Horvat E, Vaugier I, Lejaille M, et al. Comparison of three cough-augmentation techniques in neuromuscular patients: mechanical insufflation combined with manually assisted cough, insufflation-exsufflation alone and insufflation-exsufflation combined with manually assisted cough. Respiration 2014;88(3):215-222.

10. Morrow B, Zampoli M, van Aswegen H, Argent A. Mechanical insufflation-exsufflation for people with neuromuscular disorders. Cochrane Database Syst Rev 2013;(12):CD010044.

11. Mueller G, Bersch-Porada I, Koch-Borner S, Raab AM, Jonker M, Baumberger M, Michel F. Laboratory evaluation of four different devices for secretion mobilization: Acapella Choice, Green and Blue versus water bottle. Respir Care 2014;59(5):673-677.

12. Berlinski A. In vitro evaluation of positive expiratory pressure devices attached to nebulizers. Respir Care 2014;59(2):216-222.

13. Osadnik CR, McDonald CF, Miller BR, Hill CJ, Tarrant B, Steward $\mathrm{R}$, et al. The effect of positive expiratory pressure (PEP) therapy on symptoms, quality of life and incidence of re-exacerbations in patients with acute exacerbations of chronic obstructive pulmonary disease: a multicentre, randomised controlled trial. Thorax 2014; 69(2):137-143. 